\title{
Analysis of the Control Strategies of Parallel Active Power Filter
}

\author{
Limiao Huang ${ }^{1, a}$, Guiping Zhu ${ }^{1}$ \\ ${ }^{1}$ Tsinghua University, Department of Electrical Engineering, State Key Laboratory of Power System, 100084 Beijing, \\ China
}

\begin{abstract}
Depending on detection points of harmonic current, the control strategies of parallel active power filter (PAPF) can be divided into two basic types. Load current detection is the simplest open loop control strategy, but the parallel resonant problem is an inherent defect of it. Source current detection forms an effective closed loop. However, a stability problem would be caused meanwhile. In this paper, firstly, typical system of PAPF was designed and analyzed, and different kinds of current control strategies were deeply discussed. In particular, a long time ignored problem (the amplification of the fundamental current after the compensation) associated with traditional source current detection was pointed out for the first time. Next, we proposed a new double-loop compound control strategy, which detects both the load current and the source current. Through the analysis of each part of the PAPF, the mathematics model of the system was put forward. Furthermore, we used this model to discuss the stability and compensation characteristics of the system, and found out the best working point through large amount of simulation. Finally, simulation results verified the validity of the proposed control strategy.
\end{abstract}

\section{Introduction}

The parallel resonance of the grid side LCL output filter of PAPF is a serious problem of the traditional load current detection. Therefore, the compensation effect of PAPF cannot be further strengthened. Some researchers have been devoted to solve this problem from the point of current control strategies. The earliest research could be found in references [1] and [2].Authors put forward the classification criteria of the control strategies of PAPF for the first time and carried on some preliminary theoretical analysis. Then the equivalence between the two basic control strategies was proved $[3,4]$. Further, researchers deduced out the unified description of current control strategies of PAPF but without sufficient verification [4]. In reference [5], consideration has been given to both the system stability and compensation characteristics. However, the problem of the amplification of fundamental current after the compensation has been still ignored.

A typical system diagram of PAPF is shown in Fig.1. Harmonic current detection unit extracts harmonic components from the load current or source current as reference current firstly. Then, the main circuit (usually a voltage source inverter, VSI) tracks the reference current by the current tracking control unit and the drive circuit. Thus, PAPF replaces of the power grid in supplying

\footnotetext{
${ }^{\mathrm{a}}$ Corresponding author : 1372742756@qq.com
} 
harmonic current to the load to ensure that the power source current stay as undistorted sinusoidal current.

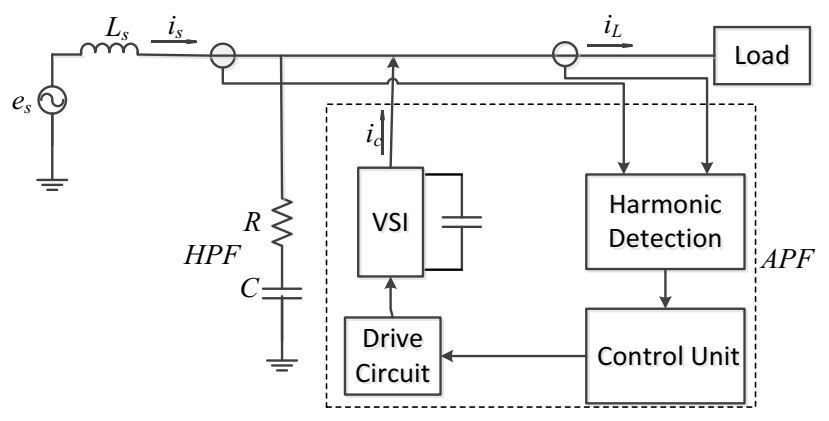

Figure 1. Typical system diagram of PAPF

As in Figure $1, i_{S}$ is the source current, $i_{L}$ is the load current, and $i_{C}$ is the output compensation current of PAPF. Assuming that $i_{L f}$ represents the fundamental component of load current, $i_{L h}$ the corresponding harmonic components. In order to eliminate the harmonic components of source current completely, we need only ensure that the compensation current be equal to the harmonic components of the load current, that is, $i_{C}=i_{L h}$.

In this paper, main circuit of the compensation current generator, a voltage type high frequency PWM inverter, was based on three phase four wire system. RC high-pass filter was connected in parallel with the main circuit in AC side to filter out high frequency harmonics of the compensation current near switching frequency. A control strategy was used to track the reference current and realize the DC side capacitor voltage control $[3,6]$. It is worth mentioning that we used FBD algorithm [7] to calculate the reference current. FBD algorithm is essentially a projection algorithm and has definite physical meaning. Its accuracy and real-time performance gain an advantage over the traditional instantaneous reactive power algorithm. Then, a three-phase diodes rectifier (with a resistance in the DC side) was served as typical nonlinear load (harmonic sources). Initial total harmonic current distortion of it reached up to $23.71 \%$ in the simulation model.

The control strategies of PAPF are usually classified by the detection points of reference current. In the following section, we made an analysis of the load current detection control strategy on the basis of the existing research results of other researchers, and pointed out the inherent defect of it. Next, math model of the source current detection control strategy and the new type of compound control strategy was put forward and discussed. Further, a problem with the source current detection (of course the same as the compound control strategy) which was neglected all the way in previous studies was pointed out, namely, the amplification of the fundamental component of source current after the compensation. Then we proposed a proper solution on the basis of theoretical analysis. Finally, we got a set of optimal control parameters of the new type of compound control strategy, which ensures PAPF to obtain best compensation result within the scope of system stability domain.

\section{Grid side output LCL filter and the parallel resonant problem}

In order to get the ideal output current, the main circuit of PAPF must be able to track the low frequency reference current accurately, with strong high frequency attenuation characteristic at the same time. This goal is closely related to the grid side output filter. LCL filter is a popular choice in recent studies. The single-phase equivalent circuit of it is shown in Figure 2. L1 is the AC side connection inductance, L2 the grid side inductance, and C the parallel capacitance, which provides 
pathway for the current harmonics around switching frequency. Some papers have formed a set of algorithm programs for the selection of specific parameters of the LCL filter [8].

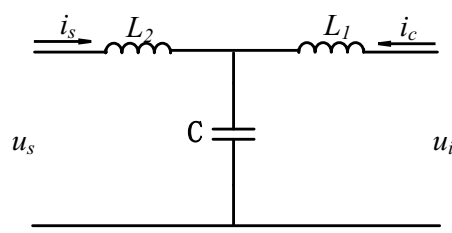

Figure 2. Single-phase equivalent circuit of LCL filter

LCL filter has excellent high frequency attenuation ability, but unfortunately, it results in the impedance resonance at the same time. The single-phase equivalent circuit of the system is shown in Figure 3. Among which, $\mathrm{R}$ is the additional damping resistance, $i_{S}$ is the source current. The sum of compensation current and load current is regarded as a current source, $i_{C L}[1]$.

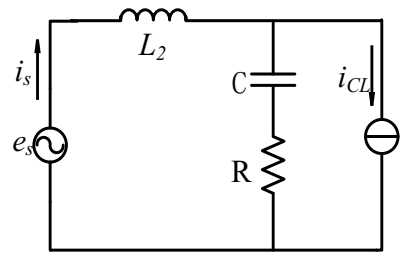

Figure 3. Single-phase equivalent circuit of the system based on LCL

Take $i_{C L}$ as the input, and $i_{S}$ the output, the transfer function of the intermediate impedance link is:

$$
G_{I}(\mathrm{~s})=\dot{I}_{\mathrm{S}} / \dot{I}_{\mathrm{CL}}=\left(R+\frac{1}{\mathrm{sC}}\right) /\left(\mathrm{sL}_{2}+R+\frac{1}{\mathrm{sC}}\right)
$$

The bode plots of it is shown in Figure 4.

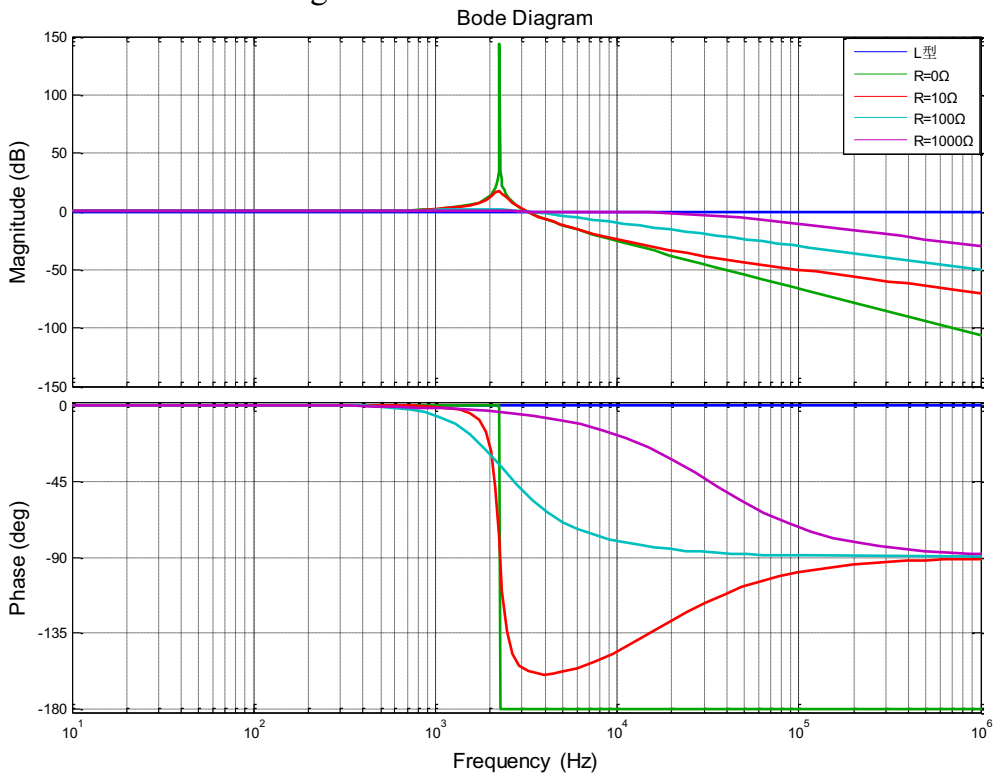

Figure 4. Bode plots of the intermediate link of impedance 
The blue line represents the traditional L filter, whose high-frequency attenuation characteristic is extremely poor. Other four lines are amplitude-frequency characteristic curves of the LCL filter under different damping resistance. As we can see, the low frequency characteristics of LCL overlap with L filter, and the high frequency attenuation reaches as high as $40 \mathrm{~dB} / \mathrm{decade}$ in the case of non-damping. However, the compensation current components near the resonance frequency (about 42 times power frequency) would be amplified before injection to the source side, and the compensation effect is seriously deteriorated. As the value of the damping resistance increasing from 0 to $1000 \Omega$, resonant peak becoming more and more small. However, the increase of damping resistance will lead to more and more system loss, and more seriously, weakening of the high frequency attenuation capability. So it is necessary to begin from the Control Strategy to regulate the system response and get good compensation result.

\section{Comparison analysis of three control strategies}

\subsection{Two basic strategies}

As has been noted, two basic current control strategies refer to the way of load current detection and the way of source current detection. The unified description of current control strategies of PAPF is deduced in reference [4]. Making use of a set of constraints and graphical method, it is not difficult to prove the equivalence between these two strategies [4]. The load current detection is the most widely used strategy. Fig.5 is the system block diagram of the PAPF. In this diagram, $G_{I}(\mathrm{~s})$ represents the transfer function of the intermediate impedance link as is shown in equation (1), and the main circuit of PAPF is equivalent to proportion link $K_{P W M}$ with time delay $T_{d}$. Because the harmonic current detection unit based on FBD algorithm which extracts the harmonic component from the input current $\left(i_{C L}\right)$ accurately in ideal situation, so it is omitted directly in Figure 5, being focused on the harmonic component current only.

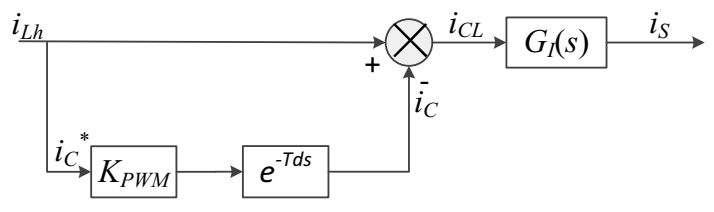

Figure 5. System block diagram of the PAPF with load current detection control strategy

Obviously, $G_{I}(\mathrm{~s})$ is outside of the closed loop, there is no way to form effective feedback to adjust it and eliminate the resonance [1]. So, we can draw the conclusion that under the way of load current detection, even if the harmonic current detection algorithm and the harmonic current tracking control achieve ideal precision and real-time performance, the compensation effect of PAPF cannot be enhanced.

In contrast, as is shown in Figure 6, an effective closed-loop feedback is formed under the way of power source current detection.

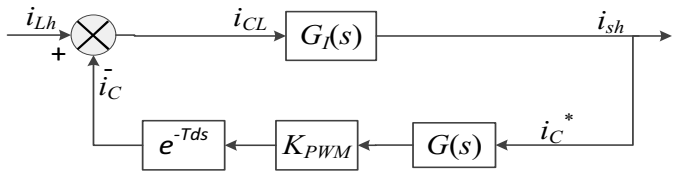

Figure 6. System block diagram of the PAPF with source current detection control strategy 
$G(\mathrm{~s})$ is the correction unit. In order to improve the system stability, $G(\mathrm{~s})$ is usually designed as a differential first-order inertia unit [5]:

$$
G(\mathrm{~s})=K T \mathrm{~s} /(T \mathrm{~s}+1)
$$

$K$ and $T$ are undecided parameters. The impedance resonance of $G_{I}(\mathrm{~s})$ can be eliminated to some extent by choosing the parameters carefully. However, because the value $K$ must be designed as large as 4 in order to acquire the best compensation effect under this way, the stability of the system would be adversely affected. Before making a further analysis on this point in the next part, we will show the compensation results of the two basic control strategies, and points out another problem which was neglected all the way in previous studies based on source current detection control strategy.

Use of three-phase uncontrolled rectifier with resistance as the harmonic source, the fundamental component of the source current $\left(\mathrm{I}_{\mathrm{f}}\right)$ is equal to $18.50 \mathrm{~A}$, and total harmonic current distortion reaches up to $23.71 \%$. The simulation results of two basic strategies are shown in Tab.1. From the table, we can see that source current detection has better harmonic control effect than load current detection. However, at the same time, the fundamental component of source current increases to $21.87 \mathrm{~A}$, a data much bigger than the original value $18.50 \mathrm{~A}$. Many studies only concentrated on the good compensation performance of the source current detection, but neglected the fact that it can also cause the amplification of the fundamental component of source current.

This paper attempts to explain this problem and suggests a solution for it in the following analysis. In general, when detection point (load side or/and source side) and detection algorithm (namely FBD algorithm in this paper) are determined, the modes of harmonic current detection could be divided into the following two categories [9].

1) Indirect mode. The fundamental component of current is calculated firstly, and its difference from the total current is taken as the output.

2) Direct mode. In this mode, the selected harmonics (of order usually less than 20) are detected directly, and the sum of them is taken as the result.

Obviously, the amount of calculation of mode 1 is much less than mode 2, so mode 1 was adopted in many applications. Inevitably, in mode 1, when the fundamental component is subtracted from total current, a small amount of fundamental component would be left in the output reference current still. Then, as the reference current passing through the correction unit G(s) which includes a amplification factor $K$ (refer to equation(2), $K$ was designed as 4 ), the remained fundamental component is amplified at the same time. This is exactly the reason that the fundamental component of the source current increases a lot after compensation. To solve this problem, we have carried on simulation research on the basis of mode 2 . The results showed that the amplification of the fundamental component of the source current was almost eliminated. Validity of the analysis was proved.

\subsection{Double-loop compound control strategy}

In practical industrial application, however, it is not the best choice to solve the fundamental current amplification problem at the expense of increasing the amount of calculation. So it is necessary to introduce our new double-loop compound control strategy. We will see in the following that the amplification factor $K$ in feedback loop of source current in this strategy could be designed far smaller than that on using source current detection strategy. Thus, mode 1 can be adopted as usual without the amplification of the fundamental component of source current.

Compound control strategy, detects both the load current and the source current as a combination of the two basic control strategies. The system block diagram of it is shown in Figure 7.

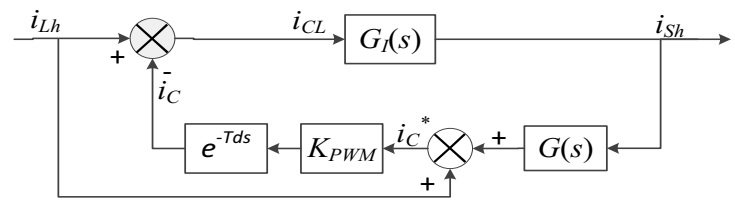

Figure 7. System block diagram of compound control strategy 
In this paper, we approximate the proportion link $K_{\mathrm{PWM}}$ as 1 and the delay of the main circuit is regarded as one PWM control cycle $\left(T_{d}=1 / f_{s w}, f_{s w}=12.8 \mathrm{kHz}\right)$. Because the switching frequency is high enough than the bandwidth of the PAPF, therefore, the delay unit could be Taylor expanded with second-order approximation.

$$
e^{-T_{d} s} \approx 1 /\left(1+T_{d} s+\left(T_{d} s\right)^{2} / 2\right)
$$

$G_{I}(\mathrm{~s})$ and $G(\mathrm{~s})$ refer to (1) and (2) respectively. Then the closed-loop transfer function of the system is expressed as follows.

$$
G_{\text {close }}=G_{I} \bullet\left(1-e^{-T_{d} s}\right) /\left(1+G \cdot G_{I} \bullet e^{-T_{d} s}\right)
$$

The characteristic equation of the system would be deduced out according to (4). Then, we can analyse the stability of the system using criterions such as the root locus rule or Routh stability criterion. In Figure 8, the square area is on behalf of the value range of $K$ and $\mathrm{T}$ that makes the system stable. The stability margin of the red area (top left corner) is larger than the other area. This shows that the smaller the parameter $K$, the better is the system stability. In the proposed double-loop compound control strategy, the reference current is mainly extracted from load current, and the source current feedback loop is just aimed to eliminate the impedance resonance. Thus, amplification factor of the source current loop (parameter $K$ ) does not need to be designed very large [5]. Therefore, the system could achieve a desired compensation effect while maintaining strong stability at the same time.

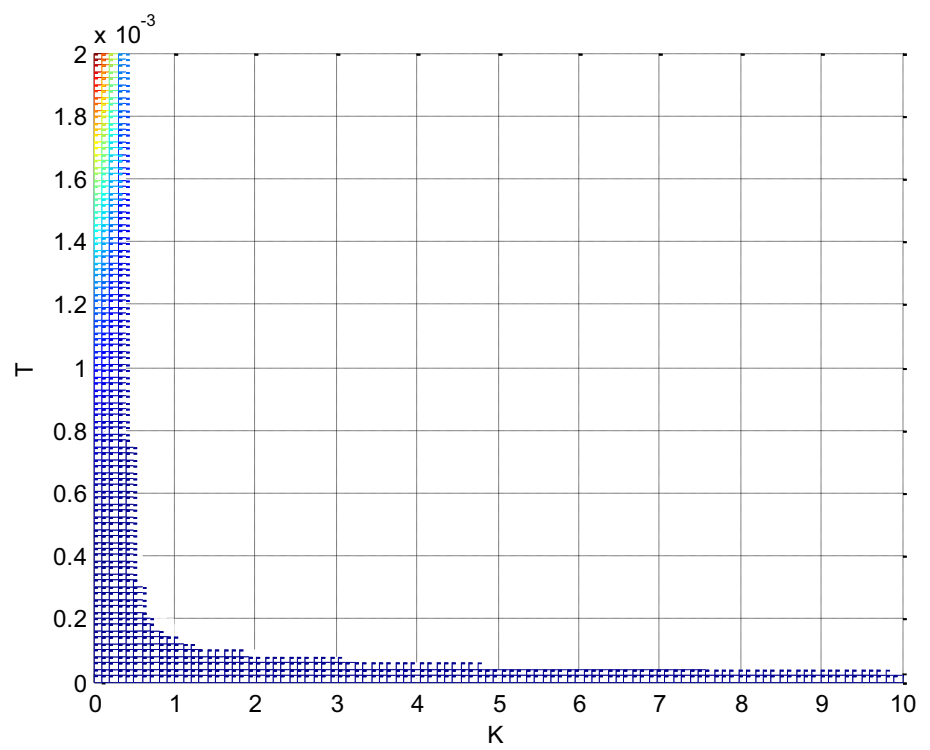

Figure 8. Value range of $\mathrm{K}$ and $\mathrm{T}$ that makes the system stable

The Comparison of the two basic strategies with the compound control strategy is shown in Table.1.

Table 1. Comparison of two basic strategies with the compound control strategy

\begin{tabular}{|c|c|c|c|}
\hline Control Strategy & $K$ & $\mathrm{I}_{\mathrm{f}} / \mathrm{A}$ & $\mathrm{THD}$ \\
\hline Load current detection & 0 & 18.97 & $4.21 \%$ \\
\hline Source current detection & 4 & 21.87 & $3.73 \%$ \\
\hline Compound control strategy & 0.2 & 19.67 & $2.89 \%$ \\
\hline
\end{tabular}

Among the three control strategies, the double-loop compound control strategy showed the most ideal compensation effect. Detailed simulation and experimental results are presented in the next section. 


\section{Simulation results}

Through the validation of a large amount of simulations, the parameter set $(K, \mathrm{~T})$ was $(0.2,0.00015)$ which made the system achieve the best compensation performance and met the condition of constraint in Fig.8 at the same time. Parameter $K$ was much smaller than 4, the value designed under source current detection control strategy.

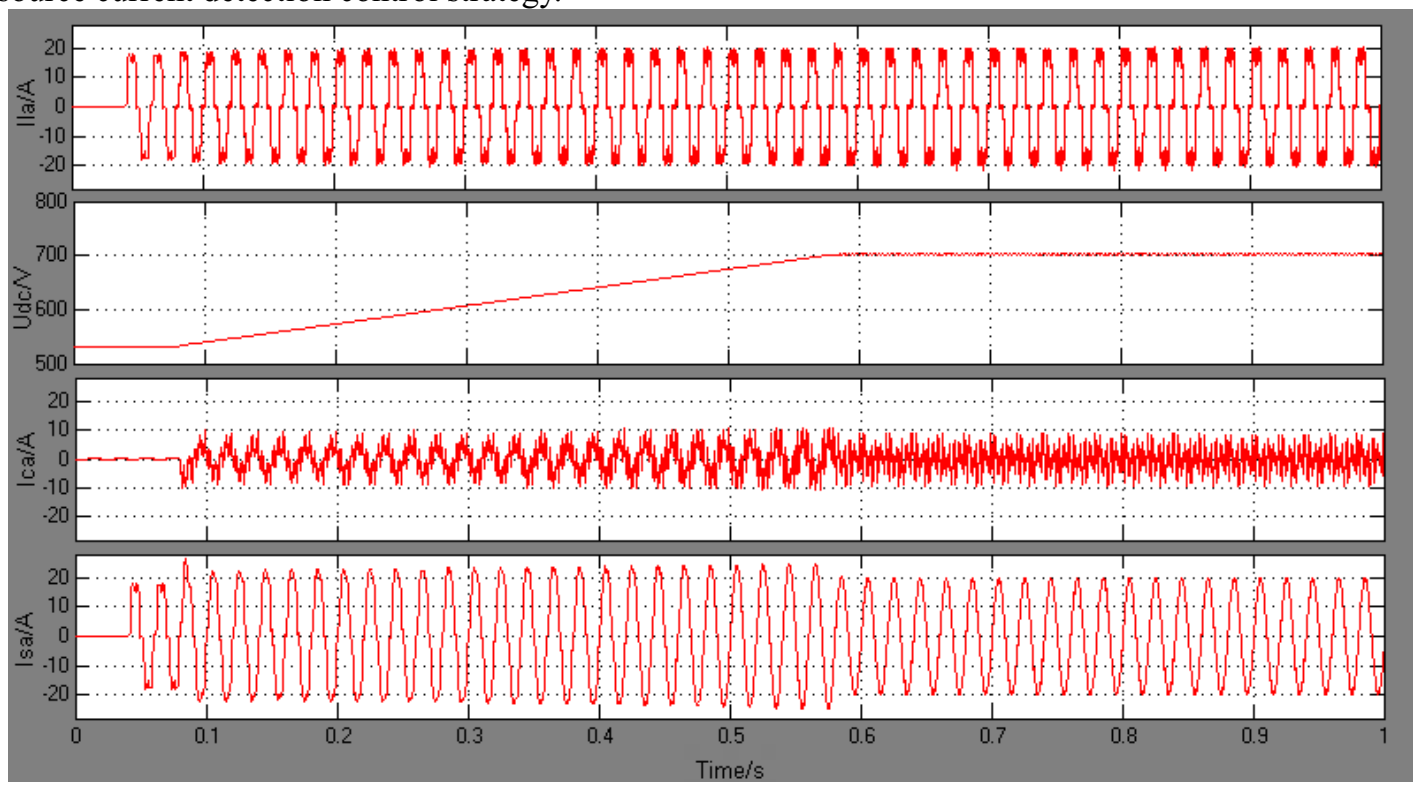

Figure 9. Simulation result under the double-loop compound control strategy

Ila: load current; Udc: DC bus voltage; Ica: compensation current; Isa: source current

Figure 9 shows one simulation result under the double-loop compound control strategy. Four curves are load current, DC bus voltage, compensation current and source current respectively. Source voltage was $380 \mathrm{~V}$, and the initial value of the DC bus voltage $\left(U_{\text {dc-start }}\right)$ was designed as $530 \mathrm{~V}$. APF soft started at $0.08 \mathrm{~s}$, and the DC bus voltage reaches its final value $\left(U_{\mathrm{dc}-\mathrm{end}}=703 \mathrm{~V}\right.$, slightly larger than the design value $700 \mathrm{~V}$ ) after 5 seconds. In this process, the reference value of DC bus voltage can be represented by:

$$
U_{\text {dc-ref }}=U_{\text {dc-start }}+\left(U_{\text {dc-end }}-U_{\text {dc-start }}\right) \cdot \mathrm{d} U
$$

$\mathrm{d} U$ is the step size of the soft start process. A voltage control loop was adopted to realize realtime tracking of this reference value. As is shown in Fig.9, the source side impact current reaches it's maximum value $27 \mathrm{~A}$ at the moment of starting APF. This suggests that the soft start design has restrained the current impacting and protected the IGBT effectively. It is worth noting that the compensation current contained a significant amount of fundamental component during $0.08 \mathrm{~s}$ to $0.58 \mathrm{~s}$. This shows that the dc-link capacitance absorbed part of active power from the source side in the process of soft start. The source current was a nearly perfect sine wave after the system reached steady state at $0.58 \mathrm{~s}$.

As is shown in Figure 10, THD of the source current after compensation is reduced from 23.71\% to $2.89 \%$. Harmonic components were mainly concentrated on the frequency band below 100 times power frequency. Switching harmonics (near by 256 times power frequency) were effectively suppressed, and the amplitude of them were smaller than 0.04A. This kind of frequency response proved the correctness of the design of the grid side LCL output filter in this study. What's more, the fundamental component of the source current equal to 19.67A, the amplification of the fundamental current under the control strategy of source current detection was well-restrained. 


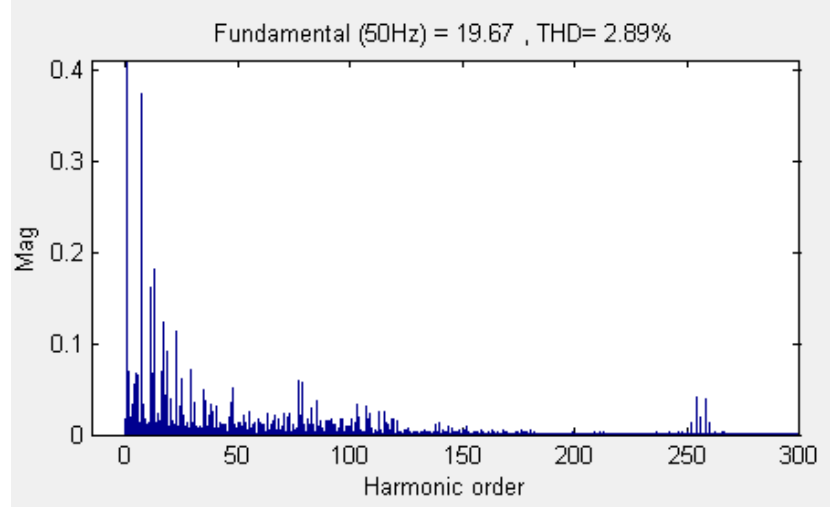

Figure 10. THD of the source current (phase A) after compensation in a simulation

\section{Conclusions}

The main work of this paper can be summarized as the following respects.

1) Designed the typical system of PAPF. Analyzed the impedance resonance problem of grid side LCL output filter and pointed out the necessity of detecting source current.

2) Built math models of different kinds of control strategies, and studied the unified description of them.

3) Pointed out and proposed a solution for the amplification problem of the fundamental current under the control way of source current detection.

4) Obtained a set of precise optimal control parameters of the proposed double-loop compound control strategy, and proved its superiorities compared with the former two basic strategies via plenty of simulations. Prototype experiments are being conducted as well, the compensation effect has to be further improved to meet the IEEE standards.

\section{References}

1. Z.A.Wang. Harmonics elimination and reactive power compensation. (China Machine PRESS, Beijing,CHINA, 2006)

2. J.Yang, Z.A.Wang. J.Xi'an Jiaotong Univ, 29(3): 97-102 (1995)

3. X.Y.Wang, J.J.Liu, C.Yuan, Z.A.Wang. IEEE PESC 2006: 1-7 (2006)

4. X.Y.Wang, J.J.Liu, C.Yuan, and Z.A.Wang. IEEE APEC 2007: 675-681 (2007)

5. X.Q.Yan, J.Yang,Z.A.Wang. Transactions of CES, 13(1): 41-45 (1998)

6. Z.Y.Yan, G.P.Zhu. Parallel Three-phase Active Power Filter Design and Development[D]. (Tsinghua Univ, Beijing, CHINA, 2012)

7. Q.Chen, J.Y.Zheng. Proc of 27th CCC: 232-235 (2008)

8. D.J.Zhang, Z.L.Qiu, Y.L.Li, G.Z.Chen, X.N.He. Transactions of CES, 26(6):137-143 (2011)

9. W.W.Liu, H.F.Ding, X.Z.Duan. Proceedings of the CSEE, 31(27): 14-20 (2011) 